\title{
Compact and Wide-band Hybrid Metal-Insulator Plasmonic Directional Coupler
}

\author{
Mahmoud Talafi Noghani and Mohammad H. Vadjed Samiei \\ School of Electrical Engineering, Iran University of Science and Technology, Tehran, 1684613114, Iran
}

Received June 20, 2014; accepted September 08, 2014; published September 30, 2014

\begin{abstract}
A compact and wideband plasmonic directional coupler is proposed based on a hybrid metal-insulator slab waveguide (HMISW). A transfer matrix method (TMM) is adopted to analyze the multilayer structure. The hybrid waveguide is composed of a metallic layer of silver and two dielectric layers of silica $\left(\mathrm{SiO}_{2}\right)$ and silicon $(\mathrm{Si})$ with high index contrast. Coupling length and maximum transferred power are optimized according to dielectric layer thicknesses. It is shown that submicron coupling length is achieved with a wide bandwidth and high efficiency, being advantageous to conventional directional couplers.
\end{abstract}

Plasmonics as a subfield of modern optics, involves physical phenomena which result in the confinement and guiding of light in metal-dielectric nanostructures. Many designs are proposed to make a better balance between confinement and loss, mainly based on three primitive structures of insulator-metal-insulator (IMI) [1-2], metalinsulator-metal (MIM) [3-4] and metal-insulator (MI) [5]. It is shown that a hybrid plasmonic structure has a better ability to provide the C-L balance [6-8]. This is a potential for realization of different components and devices based on hybrid plasmonics waveguides. Here we concentrate on directional couplers (DCs) which are utilized as power splitters, switches, modulators, etc, in many applications. Different coupling structures based on plasmonic waveguides were analyzed previously [9-13]. In this letter we propose and analyze a new directional coupler based on a hybrid metal-insulator slab waveguide (HMISW) in which coupling occurs from the metallic side. The super mode theory (SMT) is used to describe the coupling process mathematically and the transfer matrix method (TMM) is adopted to calculate propagation constants and electromagnetic fields. A hybrid directional coupler provides submicron coupling lengths with efficient power transfer $(>90 \%)$ over the whole optical telecommunication band (1260-1675nm). The proposed hybrid plasmonic slab waveguide, depicted in Fig. 1-left, is a 4-layer structure composed of a metal (Silver), low (L) index dielectric $\left(\mathrm{SiO}_{2}\right)$, high $(\mathrm{H})$ index dielectric $(\mathrm{Si})$ and cover layer (air) with constants of $\varepsilon_{A g}=-127.1-\mathrm{j} 3.46,2.1,12.1$ and 1 at $\lambda=1550 \mathrm{~nm}$, respectively. A surface plasmon wave (surface plasmon-polariton (SPP)) propagates in the $\mathrm{z}$ direction with transverse magnetic (TM) polarization.

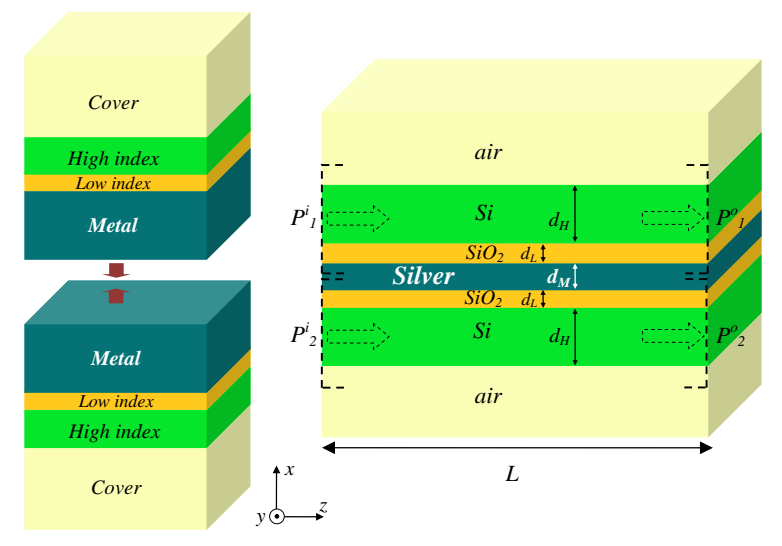

Fig. 1. Schematic of the directional coupler (right) based on a hybrid metal-insulator slab waveguide (left).

The electric field is highly confined in the L-layer due to high index contrast between $\mathrm{L}$ and H-layers. The significance of such a structure is that it could provide high confinement at a relatively low loss. It is shown that by suitably adjusting the layer thicknesses, the propagation properties of the waveguide are suitably controlled [6-8]. Figure 1-right shows a schematic representation of the proposed hybrid directional coupler. Coupling occurs when two HMISWs are brought into close proximity from the metallic side with a separation distance of $d_{M}$. According to the super mode theory, transverse modes of two totally identical lossy waveguides are classified into two groups of symmetrical and antisymmetrical modes with complex propagation constants of $\gamma_{s}=\beta_{s}-\mathrm{j} \alpha_{s}$ and $\gamma_{a}=\beta_{a}-\mathrm{j} \alpha_{a}$. The fields in each waveguide could be written as a linear combination of super modes and thus the total field is formed as a weighted sum of the waveguide fields. If we assume that the first waveguide is excited at $z=0$ with a field of $P_{i 1}$ amplitude, power coupled to the second waveguide at a distance of $z=L$ is:

$$
\begin{aligned}
P_{02}(L) & =P_{i 1}\left|\frac{1}{2}\left[e^{-\gamma_{s} L}-e^{-\gamma_{a} L}\right]\right|^{2} \\
& \cong e^{-\left(\frac{2}{L_{p}}\right) L} \cdot \sin ^{2}\left(\frac{\pi}{2 L_{c}} L\right)
\end{aligned}
$$


which is written by the assumption of $\left(\beta_{s}-\beta_{a}\right) / 2$ $>\left(\alpha_{s}-\alpha_{a}\right) / 2 . \quad L_{c}=\pi /\left|\beta_{s}-\beta_{a}\right|$ is the "coupling length" and $L_{p}=2 /\left|\alpha_{s}+\alpha_{a}\right|$ is the "mean attenuation length". Equation (1) describes the transferred power with an error of less than $1 \%$ since the phase constant $(\beta)$ is at least 2 orders of magnitude larger than $\alpha$ in hybrid structures. The transferred power decreases exponentially with a factor of $\left(\alpha_{s}+\alpha_{a}\right)$. The maximum value $\left(P_{\max }\right)$ is founded by calculating the extrema of (1) as:

$$
\begin{aligned}
& z_{\max }=\frac{2}{\pi} L_{c} \cdot \arctan \left(\frac{\pi L_{p}}{2 L_{c}}\right), \\
& P_{\max }=\frac{\exp \left(-2 x \arctan \left(x^{-1}\right)\right)}{1+x^{2}},
\end{aligned}
$$

in which $x=2 L_{c} / \pi L_{p}$. Disregarding power attenuation along the propagation direction, power conservation requires that the total power of the coupled waveguides be unity along the coupler. Thus, the power in the first waveguide $\left(P_{o l}\right)$ would be $(1)$ with the sine function replaced by cosine. A transfer matrix method is adopted to calculate complex propagation constants of guided modes following the method described in [8].

The HMISW is firstly analyzed by TMM at $\lambda=1550 \mathrm{~nm}$. The results are shown in Fig. 2. Effective refractive index curves of the HMISW for the first two TM polarized modes indicate that for H-layer thicknesses $\left(d_{H}\right)$ up to $200 \mathrm{~nm}$, the waveguide operates in a single mode region. This is true for L-layer thicknesses $\left(d_{L}\right)$ up to about $100 \mathrm{~nm}$ (detailed analysis of HMISW is provided in $[7,8]$ ). The minimum propagation lengths of the fundamental mode for $d_{L}=2,25,100 \mathrm{~nm}$ are 10,25 and $62 \mathrm{~nm}$, respectively (Fig. 2-inset). A suitable directional coupler should have the coupling length much smaller than the propagation length. The phase constant variation of symmetrical and antisymmetrical modes with respect to a change in metal thickness from 10 to $90 \mathrm{~nm}$ for $d_{L}=25 \mathrm{~nm}$ and $d_{H}=40,60$, 80, and 100nm are depicted in Fig. 3. A typical transverse profile of the E-field major component $\left(E_{x}\right)$ for the super modes is also shown in the inset. It is seen that antisymmetric modes are more sensitive to both $d_{M}$ and $d_{H}$. Obviously, maximum coupling occurs at smaller metal thicknesses where the difference between $\beta_{s}$ and $\beta_{a}$ is minimum. Figure 4 depicts the plot of $P_{\max }$ versus $L_{c}$ as a function of $d_{H}$ (10 to $200 \mathrm{~nm}$ with trajectory directions shown by an arrow) for $d_{L}=2,25$, and $100 \mathrm{~nm}$ at $d_{M}=20 \mathrm{~nm}$. The main goal in designing directional couplers is to minimize the coupling length. It is seen that the minimum $L_{c}$ for L-layer thicknesses of 2, 25 and $100 \mathrm{~nm}$ occurs at $\mathrm{H}$ layer thicknesses of 90,80 and $60 \mathrm{~nm}$ and the resulting coupling lengths are $0.46,1.17$ and $2.82 \mu \mathrm{m}$, respectively. Corresponding maximum normalized transferred powers are $0.904,0.905$ and 0.903 . Resulting values for $P_{\max }$ do not differ very much from their maximum values which are $0.910,0.911$ and 0.905 , respectively. The performance of a hybrid coupler is guaranteed since the coupling lengths of Fig. 4 are well below the propagation lengths of Fig. 2.

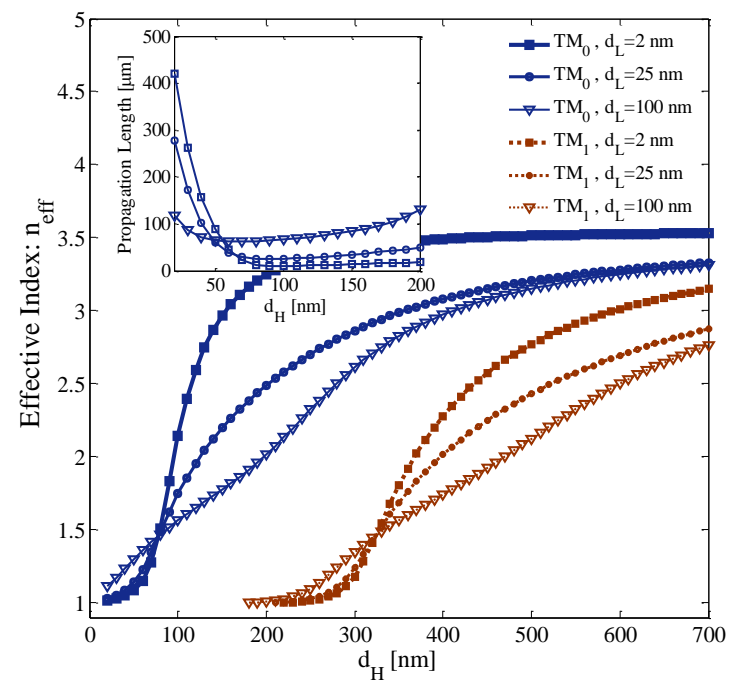

Fig. 2. Effective index of the first two TM polarized modes of HMISW, inset: propagation length of the fundamental mode $\left(\mathrm{TM}_{0}\right)$.

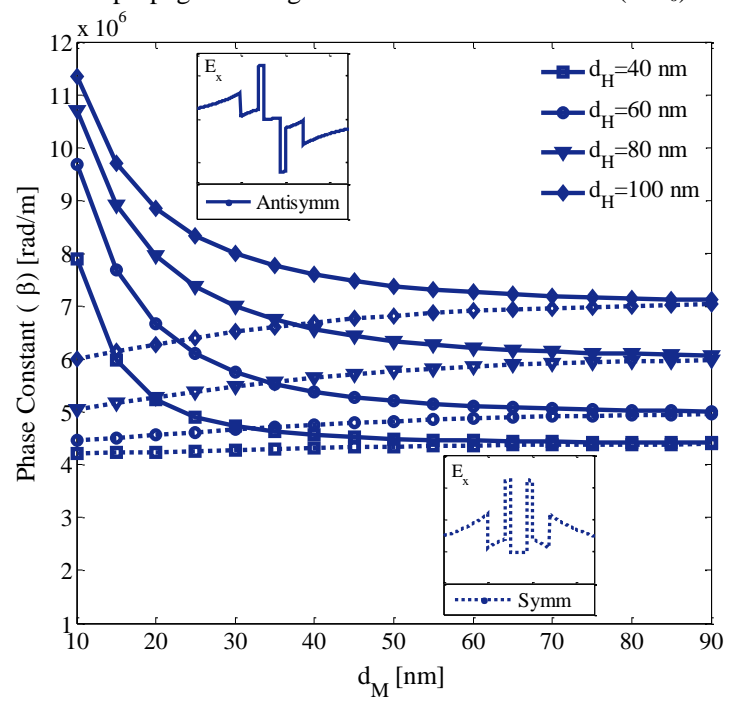

Fig. 3. Phase constant $(\beta)$ of the symmetrical and antisymmetrical modes as a function of $d_{M}$ for $d_{L}=25 \mathrm{~nm}$ and $d_{H}=40,60,80$, and 100 $\mathrm{nm}$, inset: transverse profile of the E-field major component $\left(E_{x}\right)$ for symmetrical and antisymmetrical modes at $d_{M}=70 \mathrm{~nm}$ and $d_{H}=80 \mathrm{~nm}$.

The frequency sensitivity of coupler characteristics $\left(P_{\max }\right.$ and $\left.L_{c}\right)$ is depicted in Fig. 5. The results are obtained for the whole range of optical telecommunication bands ( $\lambda=1260-1675 \mathrm{~nm})$. Optimum values of $d_{H}$ for $d_{L}=2$, $25,100 \mathrm{~nm}$ are taken into account. The coupler is reasonably stable, especially at lower thicknesses of Llayer (minimum change of $0.21 \mu \mathrm{m}$ in $L_{c}$ and 0.05 in $P_{\max }$ ). 


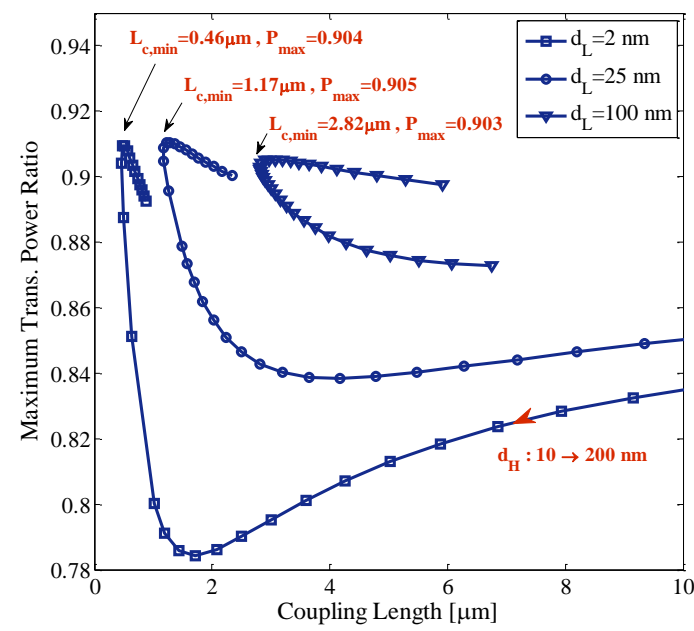

Fig. 4. Maximum transferred power ratio versus coupling length as a function of $\mathrm{d}_{\mathrm{H}}=10$ to $200 \mathrm{~nm}$ for $\mathrm{d}_{\mathrm{L}}=2,25$ and $100 \mathrm{~nm}$ at $d_{M}=20 \mathrm{~nm}$.

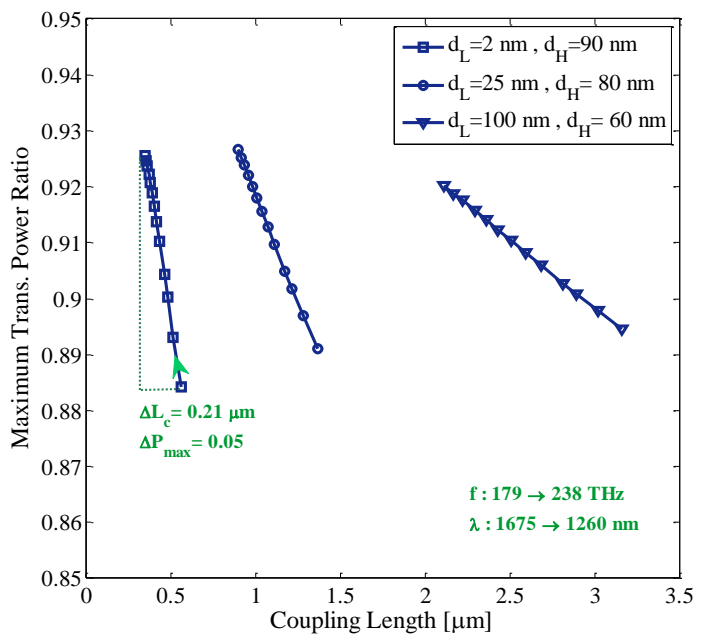

Fig. 5. Maximum transferred power ratio versus coupling length as a function of frequency: $f=179-238 \mathrm{THz}(\lambda=1675-1260 \mathrm{~nm})$ at optimum values of $\mathrm{d}_{\mathrm{H}}$ for $\mathrm{d}_{\mathrm{L}}=2,25$ and $100 \mathrm{~nm}$ at $d_{M}=20 \mathrm{~nm}$.

The performance bandwidth of the coupler is further clarified using the wavelength analysis of two dimensional versions including transition arms (length $D$ and separation distance $S$ ). In this regard, the dispersion behavior of silver is considered according to the Drude model described in [14]. Figure 6 depicts the output power frequency response for coupled waveguides at $d_{L}=10 \mathrm{~nm}, d_{H}=90 \mathrm{~nm}, d_{M}=20 \mathrm{~nm}$ and $\mathrm{L}=350 \mathrm{~nm}$ (approx. coupling length) analyzed using the COMSOL Multiphysics frequency domain simulator. The dimensions of transition arms are $D=700 \mathrm{~nm}$ and $S=200 \mathrm{~nm}$. The maximum transferred power is $-1.38 \mathrm{~dB}$ at $\lambda=1550 \mathrm{~nm}$ and varies between -1.28 and $-2.12 \mathrm{~dB}$ throughout the band. The extinction ratio $\left(\mathrm{ER}=10 \log \left(P_{\text {coupled }} / P_{\text {through }}\right)\right)$ and isolation (Iso= $\left.-10 \log \left(P_{\text {isolation }} / P_{\text {in }}\right)\right)$ are not lower than 10 and $20 \mathrm{~dB}$ through the whole band respectively, showing a very wide-band performance. ER hits a high of $23 \mathrm{~dB}$ (at $\lambda=1500 \mathrm{~nm}$ ) and Iso hits a low of $20.4 \mathrm{~dB}$ (at $1400 \mathrm{~nm}$ ). The coupler has a thickness of $420 \mathrm{~nm}$ resulting in a total dimension of about $(420 \mathrm{~nm} \times 1750 \mathrm{~nm})$ which is very advantageous in comparison to similar non-hybrid couplers [12].

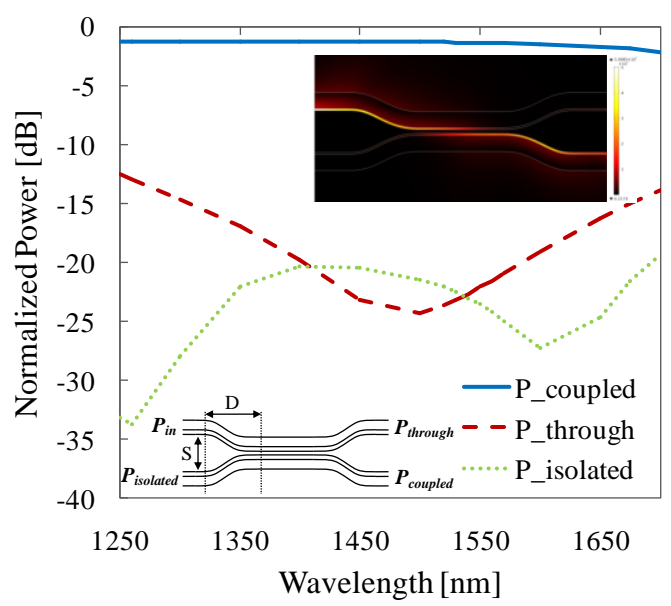

Fig. 6. Normalized power response of an HMI directional coupler as a function of wavelength at $d_{L}=10 \mathrm{~nm}, d_{H}=90 \mathrm{~nm}, d_{S}=20 \mathrm{~nm}, L=350 \mathrm{~nm}$, $D=700 \mathrm{~nm}$ and $S=200 \mathrm{~nm}$ (FEM results). Electric field distribution is shown in the inset.

In summary, a plasmonic coupler is proposed based on a hybrid metal-insulator slab waveguide. The proposed structure has the potential to develop ultra-compact and very wide-band directional couplers for applications such as splitters, combiners, switches, wavelength sorters, etc. It is shown that very short sub-micron coupling lengths $(<500 \mathrm{~nm})$ are achieved with relatively high power transfer efficiency and wide band performance (>400nm).

\section{References}

[1] P. Berini, Phys. Rev. B 61, 10484 (2000).

[2] R. Zia, M.D. Selker, M.L. Brongersma, Phys. Rev. B 71, 165431 (2005).

[3] R. Zia, M.D. Selker, P.B. Catrysse, M.I. Brongersma, J. Opt. Soc. Am. A 21, 2442 (2004).

[4] G. Veronis, S. Fan, Opt. Lett. 30, 3359 (2005).

[5] A.V. Krasavin, A.V. Zayats, Appl. Phys. Lett. 90, 211101 (2007).

[6] R.F. Oulton, V.J. Sorger, D.A. Genov, D.F.P. Pile, X. Zhang, Nature Photonics 2, 496 (2008).

[7] M.Z.F. Alam, J.S. Aitchison, M. Mojahedi, IEEE J. of Selec. Topics Quant. Elec. 19, 4602008 (2013).

[8] M.T. Noghani, M.H.V. Samiei, Springer Plasmon. 8, 1155 (2013).

[9] A. Boltasseva, T. Nikolajsen, K. Leosson, K. Kjaer, M.S. Larsen, S.I. Bozhevolnyi, IEEE J. Lightwave Tech. 23, 413 (2005).

[10] Y. Wang, R. Islam, G.V. Eleftheriades, Opt. Expr. 14, 413 (2006).

[11] H. Zhao, X.G. Guang, J. Huang, Physica E: Low-dim. Syst. Nanostruct. 40, 3025 (2008).

[12] P. Dastmalchi, N. Granpayeh, M. Rasouli Disfani, Springer Appl. Phys. A, 103, 741 (2011).

[13] M.Z. Alam, J.S. Aitchison, M. Mojahedi, Opt. Lett. 37, 3417 (2012).

[14] P.B. Johnson, R.W. Christy, Phys. Review B 6, 4370 (1972). 